Rabaska

Revue d'ethnologie de l'Amérique française

\title{
Marius Barbeau : au-delà du souvenir
}

\section{Jean-Pierre Pichette}

Volume 13, 2015

Présence de Marius Barbeau : l'invention du terrain en Amérique française. Autour d'un legs centenaire (1914-2014)

URI : https://id.erudit.org/iderudit/1033774ar

DOI : https://doi.org/10.7202/1033774ar

Aller au sommaire du numéro

Éditeur(s)

Société québécoise d'ethnologie

ISSN

1703-7433 (imprimé)

1916-7350 (numérique)

Découvrir la revue

Citer ce document

Pichette, J.-P. (2015). Marius Barbeau : au-delà du souvenir. Rabaska, 13,

372-378. https://doi.org/10.7202/1033774ar

Ce document est protégé par la loi sur le droit d'auteur. L'utilisation des services d'Érudit (y compris la reproduction) est assujettie à sa politique d'utilisation que vous pouvez consulter en ligne.

https://apropos.erudit.org/fr/usagers/politique-dutilisation/ 


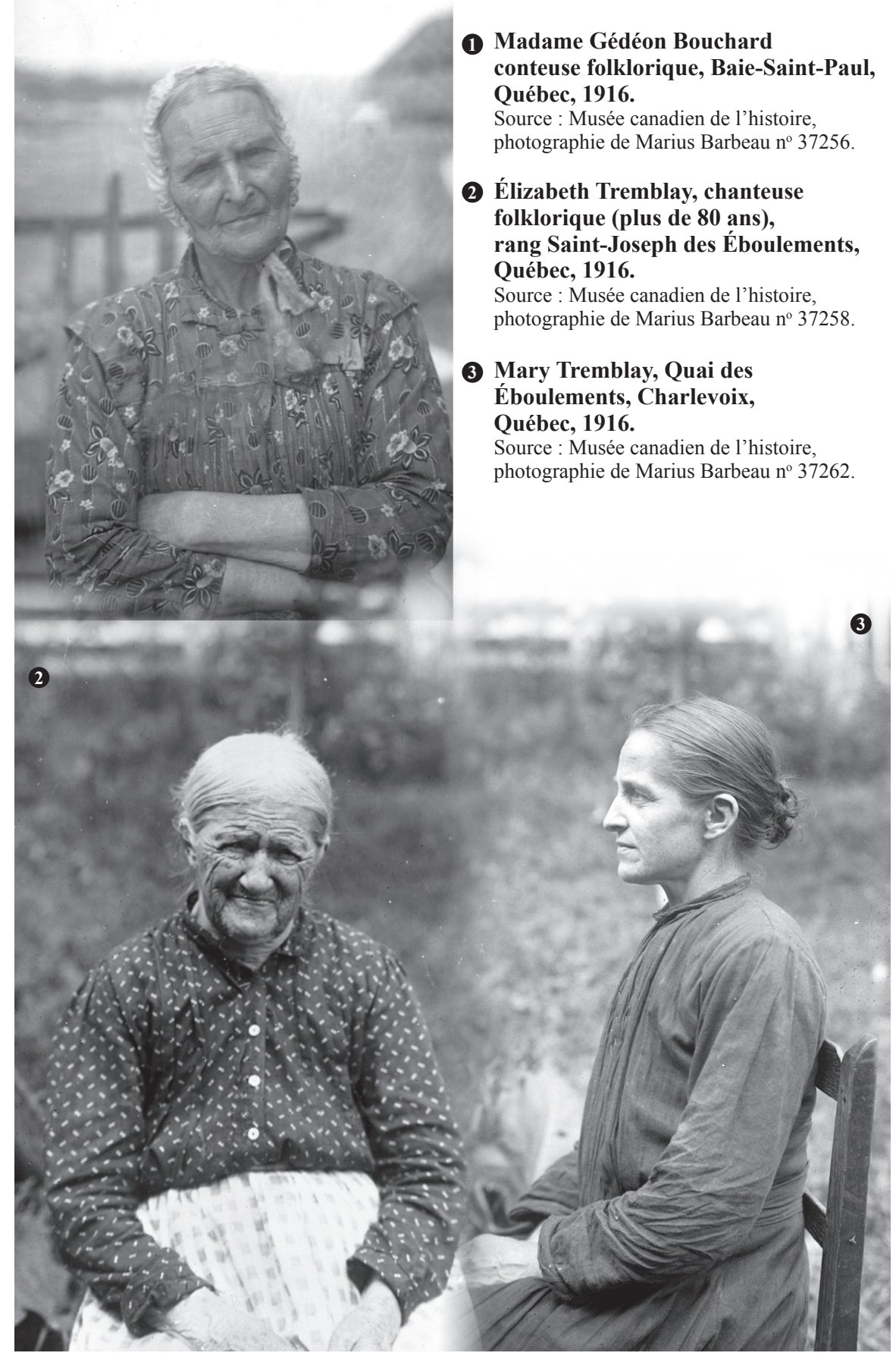




\title{
Clôture
}

\section{Marius Barbeau : au-delà du souvenir}

\author{
JeAn-Pierre Pichette \\ Société québécoise d'ethnologie \\ Professeur associé, Université Sainte-Anne
}

Personnage d'exception, Marius Barbeau laisse une œuvre immense dont on peine toujours à faire le tour. Ce colloque, dédié à quelques grands axes de son volet canadien-français, n'a voulu mettre en valeur qu'un aspect particulier de son œuvre, l'invention du terrain en Amérique française, en explorant son approche de l'enquête et sa méthode de travail, sa contribution aux domaines de l'oralité et des arts populaires, les rapports qu'il a entretenus avec les principaux collaborateurs qu'il a associés à son entreprise, son impact sur le milieu universitaire, ici et ailleurs, et finalement son actualité par son héritage, le tout placé sous le thème présence de Marius Barbeau. Plus de trente communications distribuées en une dizaine de séances, un ensemble qui soulève peut-être autant de questions qu'il apporte de réponses.

\section{La contribution de Barbeau à la discipline}

Les ethnologues d'ici savent à quel point l'entrée en fonction de Marius Barbeau comme anthropologue au Musée national d'Ottawa en 1911 a constitué un point tournant dans l'étude des traditions populaires du Canada français. Sous son impulsion, elles devenaient objets scientifiques et non plus exclusivement exercices littéraires ou curiosités touristiques. Avant lui, les littérateurs - écrivains, chroniqueurs, conteurs - du XIX siècle s'en étaient inspiré très librement pour faire œuvre littéraire ; la légende surtout leur permettait de fleureter avec l'interdit tout en le condamnant et d'échapper par ce moyen à la sanction d'une critique cléricale frileuse. Dorénavant, les folkloristes feraient des enquêtes plus ou moins prolongées auprès des dépositaires de la tradition. Comme l'a bien noté Jean Du Berger, « [1]e grand mérite de Barbeau a été de faire retour vers les conteurs ${ }^{1} »$ et les porteurs de la tradition.

1. Jean Du Berger, « Marius Barbeau : le conte et le conteur », Études fançaises, Montréal, vol. $12, \mathrm{n}^{\text {os }} 1-2$, avril 1976, p. 68. 
Plus féru de science que de littérature, Marius Barbeau ira donc aux sources de la tradition : il interroge les détenteurs eux-mêmes à qui il donne la parole et dont il enregistre très fidèlement les propos dans ses carnets de notes puis dans ses publications. Chez celui qui a parcouru Charlevoix et d'autres pays du Canada français à la recherche d'un patrimoine qu'il jugeait menacé, le terrain était l'étape indispensable de la documentation scientifique. Elle était exigeante aussi. Barbeau notait scrupuleusement les faits au rythme de la parole, à la sténographie, les observait non seulement dans leur actualité, mais dans leur profondeur, leur ancienneté, leur évolution, leur origine, leur sens. S'il consigne ses sources selon l'appareil scientifique du temps, il va même au-delà : il précise toujours le nom de ses informateurs, avec quelques détails biographiques, la date et le lieu de ses collectes, l'origine de leur information. Il ouvre ainsi la voie à l'étude savante des traditions populaires, de toutes les catégories, qu'elles relèvent de la vie matérielle, de la vie sociale ou de la vie spirituelle. Parmi les genres de cette dernière, qui inclut la littérature orale, il découvre en premier lieu le conte populaire - c'est-à-dire le véritable conte populaire, qui est récit imaginaire et intemporel, sans négliger la légende, récit de croyance d'une action fantastique ancrée dans l'histoire -; il redécouvre aussi la chanson populaire dont on croyait le répertoire épuisé depuis les Chansons populaires du Canada d'Ernest Gagnon, ouvrage paru cinquante ans plus tôt.

On le voit, le terrain tel que l'entendait Marius Barbeau n'a rien à voir avec les sondages pointus, occasionnels, ces recherches horizontales à courte vue sur un fait à la mode dans ce monde contemporain. Son objet de recherche était vraiment le monde ordinaire, le peuple disait-on alors, et son point de vue, la tradition. Il avait compris que, sans la plongée verticale dans la tradition, dans la généalogie et l'histoire de la transmission, il serait resté à la surface des choses, dans l'éphémère, tel le sondeur analysant des opinions qui n'ont plus cours le lendemain ou le lecteur de nouvelles résumant les rumeurs de la journée. La tradition oblige à contextualiser les faits observés, à les replacer dans leur milieu social, dans leur histoire, dans la psychologie et la personnalité du témoin et de sa famille. Félix-Antoine Savard décrivait ainsi le projet scientifique de Marius Barbeau : «Loin que le folkloriste soit un type d'attardé, collectionneur de ruines, il a tout au contraire, une conception biologique de sa science. Ce qui l'intéresse, c'est d'abord le fait vivant, mais, dans le fait vivant, l'élément chaînon, lié, traditionnel, et qui permettra de comprendre la profondeur humaine dans la durée. ${ }^{2}{ }$ P Pour cela, il avait le flair de dépister les bons informateurs. L'image souvent évoquée du sourcier, arpentant le terrain avec assurance à l'affût du moindre mouvement de sa

2. Félix-Antoine Savard, « Marius Barbeau et le folklore », Discours, Montréal, Fides, [1975], p. 92 . 
branche de coudrier qui le mènera jusqu'à la source, paraît assez juste pour traduire l'attitude de Barbeau ${ }^{3}$.

\section{La leçon de Longfellow ou le regard de l'Autre ${ }^{4}$}

Si d'une part Marius Barbeau se préparait bien au travail qu'il entreprenait sur le terrain, il savait aussi que le terrain le conduirait vers l'inconnu, car il avait le désir de connaître, l'appétit constant de faire surgir ce qu'il savait présent et que personne avant lui n'avait découvert : «C'est en quelque sorte la force motrice de ma vie, je crois, d'aller vers quelque chose que je sais là, quelque chose d'intéressant qui n'a pas encore été atteint. ${ }^{5} \gg$. Barbeau était un observateur attentif, qui avait compris très tôt qu'il lui suffisait simplement d'observer de l'extérieur, car le grand révélateur, c'est en définitive le regard de l'Autre. N'est-ce pas l'Autre, par sa distance et son questionnement, qui peut le mieux voir les caractéristiques d'une localité et lui renvoyer l'image qu'elle projette ? Ce qui semble vrai dans tous les cas - et que l'autochtone admet avec réticence -, c'est qu'il est plus aisé à un observateur étranger au milieu de repérer la couleur locale d'une tradition, d'en percevoir au premier abord la différence, qu'au natif pour qui elle est tellement familière qu'il ne la remarque plus. À l'inverse, il est aussi avéré que l'enquêteur de l'intérieur, désormais conscient de cette différence ou qui a expérimenté cette distance hors de son milieu, réussira mieux dans la cueillette des données que l'étranger qui n'a pas l'habitude et l'intimité que procure la longue fréquentation du milieu.

Néanmoins, le pouvoir de l'étranger, même s'il n'intervient qu'en spectateur, est bien réel. Et parfois, son impact sera colossal, déterminant même l'orientation d'une carrière et ouvrant un champ de recherche. La question de l'anthropologue germano-américain Franz Boas, «Y a-t-il au Canada des contes anciens, comme les contes de fée d'autrefois ?», posée " vers le temps du jour de l'an, à N[ew]-Y[ork] en 1914 » à un Marius Barbeau ahuri, est de cet ordre. Sa portée, en conviendra ce dernier, « devait mouler une partie de [s]on avenir » et le lancer sur une piste imprévue, qu'il avait d'avance reniée, la jugeant sans intérêt : « Mais à Lorette, écrit-il, pendant mon relevé ethnographique, je me rappelai que Prudent Sioui et sa femme avaient commencé quelques-uns de ces contes, que j'avais refusés parce qu'ils étaient

3. L'image de Marius Barbeau présenté comme « sourcier extraordinaire et travailleur inlassable » est de Luc Lacourcière, « Les Études de folklore français au Canada », Culture, vol. 6, 1945, p. 8.

4. Ce point de vue est adapté de notre article «Le Principe du limaçon ou la résistance des marges. Essai d'interprétation de la dynamique des traditions », Cahiers Charlevoix 8. Études francoontariennes, Ottawa, Société Charlevoix et Presses de l'Université d'Ottawa, 2010, p. 11-75.

5. « Les Mémoires de Marius Barbeau », enregistrés et transcrits par Carmen Roy, Ottawa, Musée canadien des civilisations, 1958, p. 49. 
trop français et d'apparence trop littéraire ${ }^{6} »$. Il retourne alors sur le terrain : « Quelques mois après cette invitation du d[octeur]r Boas, note-t-il, j'allai passer une vacance à Lorette, puis je me rendis à un conventum au Collège de Sainte-Anne, puis à la Beauce. » Ce faisant, il découvre tout un monde de culture orale qui l'avait jusque-là laissé indifférent et dont il ne soupçonnait aucunement la valeur. Partout où il passe, il interroge et il trouve. Ce jeune homme d'à peine 30 ans tourne ainsi son regard vers ses aînés, cette part de Soi qui est aussi l'Autre. Sur la piste des bons conteurs et des bons chanteurs, il se rend bientôt en Charlevoix, au Témiscouata, en Gaspésie, à la Baie des Chaleurs et à l'île d'Orléans.

Un peu comme ces touristes qui séjournent dans les centres de villégiature l'été, Barbeau passe pour un homme de la ville, mais ce fils de cultivateur se présente chez les paysans. Perçu bientôt comme un des leurs, un homme instruit certes mais qui s'intéresse à leur culture orale et la valorise, alors que trop souvent leur élite tient ce discours pour des balivernes, on l'accueille généralement bien et sa moisson est abondante.

Bien entendu, le patrimoine qu'il recueille provient de l'expérience et de la mémoire des informateurs qu'il a su dépister. Néanmoins, il est certain que, sans son intervention, la plus grande partie de ses collectes serait disparue définitivement. Quel témoin, très souvent illettré à cette époque, aurait eu l'idée et le moyen de rapporter autant de contes, de chansons, de mots et d'expressions populaires, de notes sur les métiers et les arts populaires et religieux ? Il a fallu le regard de cet étranger au milieu, de cet Autre, pour le faire. Barbeau a ainsi agi comme ces voyageurs qui remarquent la nouveauté du lieu dont ils décèlent les particularités, découvrent le paysage, les maisons, les habitants dans leur quotidien, leur langue, et qui achètent et commandent les bibelots, dessins, peintures que leurs artistes amateurs produisent pour eux. Dans les maisons de Charlevoix et d'ailleurs au Canada français, on trouvait alors probablement plus d'images saintes que de scènes de la vie locale. Encore là, c'est le regard de l'Autre qui créait cette économie touristique que l'élite locale considérait maladroite, inculte, voire insignifiante. «Peut-il sortir quelque chose de bon de Nazareth ? » (Jean 1,16), se demandait-on, incrédule, il y a deux millénaires.

Et voilà que de nos jours, ces petits riens, ces niaiseries de chansons et de contes recueillis par Barbeau et ses comparses sont devenus objets sacrés du folklore local et marqués du sceau du patrimoine culturel et immatériel du pays : « ces bagatelles n'étaient pas de pures bagatelles » comme le prétendait Charles Perrault. Ceux qui hier les rejetaient se changent aujourd'hui en gar-

6. Marius Barbeau, «En quête de connaissances anthropologiques et folkloriques dans l'Amérique du Nord depuis 1911 » (Résumé d'un cours donné à la faculté des Lettres, mars-octobre 1945), Université Laval, Archives de folklore, p. 8. 
diens de la mémoire locale. Le regard de l'Autre a changé leur propre regard sur eux-mêmes. Et dire que parfois il en est qui décrètent que, seuls, les gens du pays, les nés natifs, peuvent comprendre leur folklore. Quel retournement !

On peut donc interpréter comme décisive l'influence de Boas, ce savant étranger, sur le développement de l'ethnologie au Canada français : les centaines de contes que Barbeau nota à la sténographie dans les quelques années qui suivirent, et dont il publia un nombre important dans des revues scientifiques et des éditions populaires, sont à l'origine des grandes collectes et des études comparatives de Luc Lacourcière et de ses étudiants de l'Université Laval. Cette conséquence du regard de l'Autre n'est pas un phénomène unique : on a avancé que c'est le poème Évangéline, fruit de la vision romantique de l'Anglo-Américain Henry Wadsworth Longfellow, qui attira l'attention du monde sur le peuple acadien et qui marqua «l'éveil de la conscience collective des Acadiens », qui s'y étaient reconnus?

\section{La place des pionniers}

Le pays des gourganes, découvert par Barbeau pour la littérature orale d'abord, fut le lieu de ralliement des premiers folkloristes, Luc Lacourcière et Félix-Antoine Savard, ceux-là même qui furent les fondateurs du premier programme d'ethnologie du Québec et du Canada en 1944. Ces Archives de folklore de l'Université Laval, comme on les appelait à l'origine, maintenant septuagénaires, que le recteur de 1961 célébrait comme la Mnémosyne culturelle du Québec, auraient-elles encore l'oreille de la gouvernance de l'Université Laval? Le nom de Marius Barbeau et ceux de ses compagnons Lacourcière et Savard ont-ils encore quelque résonnance dans ces lieux de décision ? Ont-ils même leur juste part dans l'enseignement de l'ethnologie de l'Amérique française ? La question mérite d'être posée, tant la place accordée à la littérature orale - ce patrimoine immatériel par excellence - semble plus étroite à l'université qui l'a vue naître que dans les centres extérieurs. Pourtant, à considérer la popularité des conteurs et des chanteurs et le nombre croissant de festivals qui leur donnent une tribune pour se produire, le contexte serait propice à la réanimation de ce volet.

Le géant Barbeau est aujourd'hui méconnu chez nous. Pour le tirer du purgatoire où l'oubli le confine, il serait opportun d'entamer une série d'études sur ce maître dont l'envergure et la multiplicité des facettes ont jusqu'ici rebuté les chercheurs. La rédaction en français d'une bonne biographie, complète et à jour selon les règles de l'art, bénéficierait du manuscrit de ses souvenirs d'enfance et de la volumineuse transcription des mémoires qu'il a

7. Léon Thériault, «L'Acadie de 1763 à 1990, synthèse historique ", dans L'Acadie des Maritimes. Études thématiques des débuts à nos jours sous la direction de Jean Daigle, Moncton, Université de Moncton, Chaire d'études acadiennes, 1993, p. 55. 
confiés à Carmen Roy en $1958^{8}$. Aussi, par une plongée dans les volumineuses archives déposées au Musée canadien de l'histoire, à l'Université Laval ou ailleurs, par l'examen des écrits divers de ce polygraphe, de ses publications savantes ou populaires sous forme d'articles et de livres - dont Clarisse Cardin dénombrait 22 inédits et 578 imprimés en 1946, un quart de siècle avant sa disparition -, on saisirait avec plus de rigueur son cheminement intellectuel, le choix de ses champs de recherche, son appétit de connaître et de mettre en valeur ses découvertes. De même, le dépouillement de sa substantielle correspondance - estimée à plus de 67000 lettres reçues et envoyées à quelque 5400 correspondants $^{9}$ - dégagerait l'étendue de son réseau tentaculaire de collaborateurs au Québec, au Canada et à l'international, et permettrait de mieux apprécier l'influence intellectuelle qu'il a exercée de son vivant et qui se répercute peut-être encore de nos jours. Peut-on, par exemple, lui attribuer le retour sur le terrain de jeunes chercheurs passionnés alors que les modèles ont été plutôt clairsemés depuis une trentaine d'années ? Le corpus complet des contes et récits légendaires qu'il a recueillis, ses premiers travaux dans le domaine canadien-français, mériterait une édition scientifique à part, comme bien d'autres sujets qu'il a touchés durant sa longue et riche carrière. Souhaitons que la publication des actes de cette rencontre internationale ranime le désir de reconnaître et de mesurer la contribution de Marius Barbeau, et soit l'amorce d'études sur le père de l'ethnologie en ce pays.

$* * *$

\section{Remerciements}

En terminant, il reste à remercier les personnes qui ont collaboré de près à la tenue de ce colloque : les membres du comité scientifique et organisateur d'abord - Messieurs Serge Gauthier, du Centre de recherche sur l'histoire et le patrimoine de Charlevoix, et Christian Harvey, de la Société d'histoire de Charlevoix, qui en ont été les initiateurs, les artisans et les hôtes ; Messieurs Jean Simard et Bertrand Bergeron, de la Société québécoise d'ethnologie et de la revue d'ethnologie de l'Amérique française Rabaska, qui ont été des conseillers avisés dans la programmation de ce colloque et dans la publication des actes - ; ainsi que tous les intervenants dont les communications forment la matière de ce volume. Merci enfin aux organismes subventionnaires dont les noms figurent dans les pages liminaires, notamment le Conseil de recherche en sciences humaines dont la subvention a assuré la réalisation de cet événement.

8. «Les Mémoires de Marius Barbeau », op. cit. Ces manuscrits comptent plus d'un millier de pages.

9. Données fournies par Benoît Thériault, archiviste au Musée canadien de l'histoire. 